\title{
ALMORZANDO EN UN BANCO EN SANTIAGO DE CHILE: ANÁLISIS DE LA ORGANIZACIÓN Y REGULACIÓN SOCIAL DE LA COMENSALIDAD INSTITUCIONAL ${ }^{1}$
}

\section{LUNCHING IN A BANK OF SANTIAGO DE CHILE: ANALYSIS OF THE ORGANIZATION AND SOCIAL REGULATION OF THE INSTITUTIONAL COMMENSALITY}

\author{
Benjamín Concha González* \\ Juan Alfaro Figueroa** \\ Claudia Giacoman Hernández ${ }^{* * *}$
}

RESUMEN

\begin{abstract}
Este artículo explora las prácticas de comensalidad de los empleados que almuerzan en un banco en Santiago de Chile, con el fin de comprender y caracterizar la forma en que se desarrolla la comida compartida en contextos laborales. El trabajo de campo se realizó principalmente durante el mes de julio de 2015 e incluyó observaciones y entrevistas informales. Los resultados evidencian que las prácticas de comensalidad institucional están fuertemente normadas y modeladas por el contexto organizacional.
\end{abstract}

PALABRAS CLAVE: CHILE * SOCIOLOGÍA LABORAL * TRABAJADOR * AMBIENTE DE TRABAJO * CULTURA ORGANIZACIONAL

$1 \quad$ Este artículo es parte de los resultados del proyecto Comisión Nacional de Investigación Científica y Tecnológica (CONICYT), Fondo de Desarrollo Científico y Tecnológico (Fondecyt) de Iniciación $\mathrm{N}^{\circ}$ 11140407. Para revisar resultados obtenidos por publicaciones anteriores de este proyecto, revisar Giacoman, C. (2016). The dimensions and role of commensality: A theoretical model drawn from the significance of communal eating among adults in Santiago, Chile. Appetite, 107, 0195-6663, pp. 460-470; Giacoman, C., Leal, D., y Rivera, V. (2017). Daily rhythms of eating in Santiago, Chile. British Food Journal, 119 (6), 0007-070X, pp. 1189-1201.

* Pontificia Universidad Católica de Chile. baconcha@uc.cl

** Pontificia Universidad Católica de Chile. jialfaro@uc.cl

*** Pontificia Universidad Católica de Chile. cgiacoma@uc.cl 


\section{ABSTRACT}

This article explores the commensal practices of employees who have lunch at a bank in Santiago, Chile, in order to understand and characterize the way in which shared food is developed in work contexts. The fieldwork was carried out mainly during the month of July 2015 and included observations and informal interviews. The results show that the practices of institutional commensality are strongly regulated and modeled by the organizational context.

KEYWORDS: CHILE * OCCUPATIONAL SOCIOLOGY * WORKER * WORK ENVIRONMENT * ORGANIZATIONAL CULTURE

\section{INTRODUCCIÓN}

Analizar el fenómeno de la alimentación desde una perspectiva sociológica supone reconocer que además de las necesidades biológicas de supervivencia que se satisfacen al comer, existen también prácticas, representaciones $y$ normas que estructuran la alimentación. Es así como el imperativo fisiológico de alimentarse es también una actividad común al género humano, a través de la cual los procesos de socialización e interacción pueden ser estudiados (Simmel, 1986).

Una de las características fundamentales de la alimentación refiere a la comensalidad, entendida como la práctica de comer con otras personas (Sobal, Bove y Rauschenbach, 2002). La comensalidad reconoce la existencia de barreras que delimitan la exclusión o inclusión de comensales, regulaciones que se deben emplear en un determinado contexto social y las jerarquías internas que subyacen a los intercambios simbólicos y comunicacionales que ocurren entre los que participan en esta (Douglas, 1972). De hecho, en distintas sociedades, las formas de comer solitarias son normativamente percibidas como impropias, atípicas $y$ desestructuradas con respecto a las obligaciones sociales inherentes a comer en compañía (Grignon, 2001).

El presente artículo está enmarcado en el estudio de la comensalidad en su expresión institucional, esto es, el tipo de comensalidad que acontece en el contexto de una institución en particular, siendo esta determinada por las jerarquías, tiempos, espacios, grupos, géneros y edades que la conforman. Además, la literatura es escasa, especialmente en América Latina. Esto puede deberse a la poca valoración hacia la alimentación como un fenómeno social. Por lo tanto, el objetivo de este artículo es explorar las prácticas de comensalidad de las personas empleadas que almuerzan en un comedor institucional, para lo que se eligió el caso de un banco en Santiago de Chile, que reunía condiciones para analizar el fenómeno estudiado de manera más intensa.

El trabajo de campo se realizó a partir del uso de técnicas cualitativas, principalmente durante el mes de junio de 2015. Esto debido a la posibilidad de observar de primera fuente las actividades llevadas a cabo por los comensales. Concretamente, se efectuaron observaciones en el comedor del banco, entrevistas informales con miembros del área de recursos humanos (RR.нн.) y la revisión de material documental de la organización, así como de la legislación vigente sobre este tema. De este modo, se puede dar cuenta de las prácticas de comensalidad cotidianas, comprendiendo además el funcionamiento de la hora del almuerzo en el banco.

El trabajo de campo realizado evidencia que las prácticas de comensalidad institucional se encuentran fuertemente normadas $y$ modeladas por el contexto organizacional, pues la manera en que se desarrolla refleja las reglamentaciones y jerarquías de la institución, a la vez que se manifiestan convenciones sociales vinculadas con los ritmos alimentarios y las maneras de mesa.

El estudio de la comensalidad es relevante en tanto se pueden identificar interacciones, normas y divisiones que intervienen en 
las reglamentaciones que estructuran la vida social (Giacoman, 2016). Esto reviste especial interés, considerando que la comensalidad institucional permite explorar formas específicas de sociabilidad y comunicación que se manifiestan en el espacio laboral (Kniffin et. ál, 2015). Este trabajo permite así tener una primera aproximación a la forma en que se desarrolla la comida compartida en contextos laborales y los elementos que la caracterizan desde una perspectiva sociológica.

En Chile, las investigaciones sobre alimentación y bienestar laboral se han desarrollado desde las ciencias de la salud, enfatizando los estados nutricionales de la población trabajadora, hábitos alimentarios, prevalencias de enfermedades en la fuerza laboral y los efectos de la ingesta calórica en la productividad. Esto muestra la falta de investigación respecto al funcionamiento de las instituciones, la interacción entre las personas empleadas y los límites reflejados en la cotidianeidad de la alimentación.

Frente a la escasa investigación nacional e internacional relacionada a la comensalidad laboral, este artículo espera contribuir a la temática en cuestión, abordando el comedor institucional de un banco como una arena cultural donde se despliegan formas de sociabilidad, transmisiones de información, actualizaciones del orden institucional interno $y$ un entendimiento práctico en torno a la forma de comportarse en la mesa. Estudiar este tema en Chile también resulta relevante debido a la gran cantidad de horas que los chilenos pasan en sus trabajos y la consiguiente necesidad de alimentarse durante la jornada.

Según el último estudio realizado por la Organización para la Cooperación y el Desarrollo Económico (ocDe, 2017), de 38 países analizados, Chile es el quinto país que más horas en promedio trabaja anualmente, incluso por sobre el promedio total de la muestra de países contemplados en el estudio (1988 y 1766 horas anuales en promedio por trabajador respectivamente). Por su parte, en la Primera Encuesta Nacional de Empleo, Trabajo, Salud y Calidad de Vida de los Trabajadores y Trabajadoras en Chile (ENETS), los datos muestran que entre los trabajadores $y$ las trabajadoras, 80\% dice desayunar, 89,9\% señaló contar con horarios establecidos para almorzar y $76 \%$ manifestó tener un lugar destinado para la colación (ENETS, 2009).

A su vez, el estudio piloto a nivel mundial realizado por la Organización Internacional del Trabajo (огт), en Chile en el año 2012, se logró recabar información inicial de caracterización a partir de una muestra nacional no aleatoria: $61 \%$ de los encuestados recibe algún beneficio de alimentación, $22 \%$ no dispone de un lugar para comer, $30 \%$ de los encuestados declara no almorzar regularmente. También, la mayoría de los encuestados dispone de 45-60 minutos para almorzar y en micro, medianas y grandes empresas la mayoría declara que la forma óptima de recibir alimentación en el trabajo es mediante un comedor (orT, 2012). Lo anterior apoya la tesis de que el espacio de alimentación institucional es relevante dentro de la rutina alimentaria de los chilenos, dando la opción de tener un momento de sociabilización.

En el siguiente apartado, se presenta una revisión de la literatura en ciencias sociales sobre la alimentación en el espacio laboral, para luego proseguir con una explicación sobre la metodología utilizada en este trabajo. Los resultados obtenidos son expuestos en dos partes: ella primera se dedicada a la descripción del contexto organizacional y legal que enmarca la comida realizada en el banco; la segunda describe las prácticas concretas de comensalidad que se dan en el comedor investigado. Finalmente, se exponen las conclusiones obtenidas a partir de los principales hallazgos de la investigación.

\section{LA LITERATURA SOBRE ALIMENTACIÓN Y COMENSALIDAD EN EL TRABAJO}

El trabajo y la alimentación son actividades centrales de la vida social en la medida que se encuentran en la base de su producción y reproducción, así como en su delimitación temporal. Sin embargo, dado el poco tiempo que lleva desarrollándose la sociología de la alimentación en el continente, aún son pocos los estudios en ciencias sociales que se han dedicado a analizar en profundidad la relación entre estos dos fenómenos. 
Para las personas que trabajan, gran parte de las comidas que efectúan fuera del hogar corresponden a aquellas que realizan durante la jornada laboral. Dada la relevancia del trabajo en la vida cotidiana, la alimentación en contextos laborales adquiere centralidad en las sociedades contemporáneas ${ }^{2}$. En vista de esto, generalmente se comparte más tiempo con los compañeros y las compañeras de trabajo que con la familia o los amigos y se come frecuentemente con ellos, tal como señala Heynig (2015) en su investigación.

Comer en el trabajo representa una ruptura dentro de la jornada laboral que implica la coordinación entre los ritmos productivos $y$ los ritmos alimentarios, regulados socialmente ${ }^{3}$. En Chile, el Código del Trabajo (2016b) también contempla el derecho a tener un horario para alimentarse durante la jornada laboral. Tal como señalan autores que estudian la vida cotidiana, la comida en el trabajo es una práctica cuya realización va a implicar una espacialidad y temporalidad, debido a que demanda un lugar y un tiempo determinado para ser efectuada.

Las comidas realizadas durante el horario de trabajo pueden efectuarse, tanto en restaurantes comerciales fuera de la organización como en comedores institucionales que proporcionan comida o incluso, puede ser llevada al trabajo por las personas empleadas para ser consumida en lugares establecidos para dicha actividad o para ser ingerida en los mismos puestos de trabajo.

Distintos autores coinciden en señalar que las comidas realizadas en el trabajo son importantes instancias de sociabilidad entre compañeros y compañeras. Habitualmente, las personas tienden a compartir las comidas entre aquellos que tienen una labor similar

2 Véase Mestdag, I. (2005). Disappearance of the traditional meal: Temporal, social and spatial destructuration. Appetite, 45, pp. 62-74.

3 Aymard, M., Grignon, C., y Sabban, F. (1993). A la recherche du temps social. En Aymard, M., Grignon, C., Sabban, F., Le temps de manger. Alimentation, emploi du temps et rythmes sociaux, Paris: Éditions de la Maison des sciences de l'homme, pp. 1-40. $y$ un estatus equivalente en la institución; no obstante, ocasionalmente se puede comer con las jefaturas o subordinados (Giacoman, 2016). La comensalidad que se da en el trabajo es del tipo institucional (Grignon, 2001), la cual se caracteriza por estar jerarquizada $y$ determinada según las normas de la institución. Pero la sociabilidad producida no depende exclusivamente de la institución, pues al momento de sociabilizar se traen elementos del hogar, tal como rescatan Lindén y Nyberg (2009).

Además, diversas investigaciones han concluido que se genera una mayor unión entre los miembros de un grupo laboral gracias al encuentro y comunicación que se produce entre las personas empleadas al momento de comer, aunque también estudios indican que la comida con compañeros y compañeras de trabajo con los que hay malas relaciones resulta conflictiva (Giacoman, 2016).

La comensalidad, y por lo tanto también aquella de tipo laboral, se puede analizar a partir de tres dimensiones (Giacoman, 2016): la interaccional, la simbólica y la normativa. Dada la naturaleza de este estudio, se profundizará en la primera dimensión (de todos modos, se abarcarán las tres dimensiones en el análisis), la cual está marcada por la copresencia de los comensales y la comunicación que se produce entre ellos (Giacoman, 2016).

Dicho lo anterior, en la literatura se encontraron características propias de la comensalidad laboral que forman parte del contexto en que se lleva a cabo, tales como: con quién se come, el tiempo y el lugar. La pregunta con quién se come ha sido trabajada por Heynig (2015) en un hospital en Santiago de Chile, concluyendo que se dan tres tipos de comensales: los que siempre comen juntos, aquellos que comen solos y quienes no tienen un patrón establecido (por ejemplo, debido a la carga laboral), por lo que comen con otra persona dependiendo de la situación. Al respecto, vale mencionar que en el trabajo no siempre se puede elegir con quién se va a comer. En este caso, los turnos fijos y comunes son un facilitador de dicha coordinación. Una posible hipótesis para este fenómeno sería la mantención 
de las posiciones dentro de la escala laboral, respetando los cargos y reforzando las barreras de interacción.

En efecto, los turnos de trabajo es un elemento para considerar. Es decir, en diferentes turnos de trabajo se pueden producir momentos de alimentación distintos (por ende, de comensalidad) dado el uso de comedores, o las diferencias entre traer comida desde la casa o comprar en el lugar de trabajo. Por ejemplo, Waterhouse, et. ál., (2003) encontraron en su investigación diferencias en la alimentación de comida fría o caliente según los turnos en el trabajo. También se producen cambios en la relevancia del comedor como lugar de comida, resultado encontrado por Stewart y Wahlqvist (1985). Sin embargo, el número de comidas dentro del trabajo no se vería afectado según el turno (Takagi, 1972).

Otro elemento relevante que considerar en la comensalidad institucional son las diferencias de estatus al interior de la organización. La jerarquía es un elemento importante a la hora de elegir con quién comer y qué comer, dado que entre compañeros y compañeras del mismo rango se puede conversar de temas de interés común. Esto aporta en el proceso de conformación de grupos de sociabilización basados en la comensalidad. Por otra parte, comer con personas desagradables puede llevar a que los comensales coman más rápido o incluso, que alguno coma solo como forma de evitarlo. En el caso de comer con alguien de otra jerarquía, se menciona la posible expresión de tensiones (Giacoman, 2016).

En la investigación de Heynig (2015) se constató que la mayoría de la población trabajadora del hospital comían con personas que ocupaban un puesto similar en la organización. Siguiendo la misma línea, la conformación de grupos de comensales se puede ver influida por la semejanza y la diferenciación entre los pares trabajadores.

Ahora bien, este espacio de alimentación se ha vuelto problemático debido al uso del tiempo, produciendo en muchos casos una sensación de agobio en los trabajadores y las trabajadoras. Esto se puede manifestar en la percepción de que el tiempo no alcanza y hay que buscar formas de coordinación para comer con los compañeros y compañeras.

Junto con estos elementos, el lugar es otra dimensión contextual que ayuda a explicar el fenómeno de la comensalidad laboral. Como señala Valentine (2002), el lugar de trabajo se construye en la interacción de la población trabajadora con los elementos materiales y no materiales del lugar. En la literatura revisada se ha observado como resultado que el espacio es un elemento importante para facilitar la sociabilización entre estos. Esto es relevante, dado que el espacio en el cual se decide comer condiciona las relaciones que se establecen con los compañeros y las compañeras de trabajo, lo cual también aporta en la construcción de identidad de un grupo.

En el trabajo de Lindén y Nyberg (2009) se resalta el comedor como un espacio para conocer a personas compañeras de trabajo, incluso en un ambiente de interculturalidad $y$ en un trabajo solitario, como lo es el de conductor de buses urbanos. Estos autores observan que en el comedor se daba la posibilidad de compartir ingredientes de cocina, así como distintos platos que eran traídos desde la casa. Por su parte, el estudio de Kniffin et. ál., (2015) observa que el lugar para comer es un espacio de sociabilización, que facilita una mejor colaboración entre la población trabajadora. Un último hallazgo que es necesario profundizar, es la diferenciación simbolizada a partir del lugar de alimentación, lo cual es un importante resultado obtenido por Thelen (2006). Esto ayuda en la investigación respecto a la conformación de grupos de comensales a partir de características comunes entre ellos.

En síntesis, la literatura sobre alimentación en el trabajo y específicamente sobre la comensalidad en este espacio es aún escasa. Pese a ello, existe consenso en identificar que la comida junto con los compañeros y compañeras de trabajo es central en las relaciones sociales establecidas entre las personas empleadas y un elemento relevante en la delimitación de los ritmos de la jornada laboral. 


\section{METODOLOGÍA}

El análisis realizado para el presente artículo se enmarca en la primera fase cualitativa de un proyecto de investigación mayor cuyo objetivo es describir las prácticas y valores relativos a la comensalidad en adultos de la Región Metropolitana de Santiago. Para explorar las prácticas de comensalidad institucional, se optó por estudiar el caso del comedor de un banco como un primer acercamiento. Esto dada las facilidades entregadas por dicha institución para realizar la investigación. También, algunos sociólogos consideran que la civilización de la oficina está asociada a nuevos patrones de alimentación en la población (Fischler, 1995). En este sentido, el estudio de la comensalidad dentro de un banco es un ejemplo de civilización de la oficina, ya que trabajadores y trabajadoras de diferentes jerarquías se reúnen en un espacio común (el casino), lo cual puede presentar signos de distinción. Debido a que esta era una primera exploración al tema, se optó por realizar un trabajo de campo breve, pero que permite insertarse en el momento de comensalidad.

El banco analizado es una organización de carácter privado e internacional que opera en más de 30 países y que está presente en todo el territorio nacional. En Chile cuenta con 3739 empleados, de los cuales $46 \%$ son hombres $y$ $54 \%$ son mujeres. En la casa matriz del banco -donde se realizaron las observaciones- trabajan 636 personas actualmente. Este banco fue considerado un caso pertinente para realizar un trabajo de campo exploratorio, debido a que reunía condiciones que lo hacían un buen lugar para observar la comensalidad institucional descrita por autores como Grignon (2001): el banco contaba con un comedor al que podía acceder únicamente los miembros de la institución y las personas trabajadoras disponían un tiempo asignado en su jornada para almorzar.

Durante el transcurso de 4 semanas -comenzando el 13 de julio y finalizando el 7 de agosto de 2015- se estudiaron las prácticas de comensalidad que acontecen en el banco. El ingreso a la institución fue posible gracias a un empleado del banco que actúo como portero, quien luego de ser contactado, coordinó una reunión con la encargada de RR.HH., instancia en la cual se explicó el objetivo del estudio, el compromiso de anonimato con el banco y donde se hizo entrega de un consentimiento informado que señalaba la voluntariedad de su participación. Utilizando la técnica de observación no participante, se realizaron observaciones en distintos días, horarios y mesas del comedor, con el fin de registrar diferencias que pudieran existir en los asistentes que estaban almorzando en esos momentos de la jornada laboral. Las observaciones se extendían por alrededor de 3 horas, tiempo durante el cual se tomaba notas de campo que luego fueron transcritas a un computador y codificadas temáticamente mediante el software cualitativo MAXQDA.

Se optó por un diseño de investigación exploratorio debido a la escasa información académica relativa a la comensalidad institucional en Chile. Además, se optó por la observación no participante, puesto que es una técnica que permite insertarse en el espacio social estudiado, resguardando no perturbar la rutina diaria de los asistentes al comedor.

La información registrada en las notas de campo se complementó con la revisión de material documental —específicamente reportes anuales de sustentabilidad entre los años 2013 y 2015- así como, 3 entrevistas informales con miembros del área de RR.Hн., con el fin de obtener una triangulación que contextualizara institucionalmente los datos observacionales. También se revisó la legislación vigente en Chile que enmarca las prácticas de alimentación en el trabajo del banco estudiado.

\section{RESULTADOS}

La exploración de las prácticas de comensalidad institucional en el banco estudiado muestran que esta se encuentra en gran medida modelada por las regulaciones formales e informales, siendo las primeras propias de la institución y el marco legal en el que se inserta (Grignon, 2001), mientras que las segundas corresponden a convenciones sociales sobre la alimentación correcta y las maneras de llevarla a cabo (Fischler, 1995). En consecuencia, se presentarán los resultados obtenidos a partir de dos aspectos de la comensalidad en el banco: primero, la comensalidad en el marco 
del contexto institucional y sus regulaciones; segundo, las prácticas efectivas de comensalidad y las convenciones sociales que se manifiestan.

\section{1) EL CONTEXTO INSTITUCIONAL} DE LA COMENSALIDAD

En promedio, las personas trabajadoras chilenas pasan 44,8 horas semanales en el trabajo (cantidad levemente mayor a las 43,4 horas promedio a la semana del sector financiero) (Departamento de Estudios Dirección del Trabajo, 2014). Por otra parte, el Código del Trabajo establece normas y estándares relativos a la alimentación que el empleador debiese cumplir, por ejemplo, que, con excepción de algunos tipos de trabajos, "la jornada de trabajo se dividirá en dos partes, dejándose entre ellas, a lo menos, el tiempo de media hora para la colación. Este período no se considerará trabajado para computar la duración de la jornada diaria" (Dirección del Trabajo, 2016b, p. 38). Asimismo, la interpretación jurídica del artículo 34, señala expresamente que el objetivo propuesto por la ley no es otro que "el consumo de un alimento o comida ligera, necesaria para reparar las fuerzas gastadas durante la primera parte de la jornada diaria" (Dirección del Trabajo, 1996, p. 3). Por otra parte, un contrato de trabajo entiende la alimentación como un beneficio adicional y la colación no constituye remuneración (Dirección del Trabajo, 2016a).

Teniendo en cuenta la legislación nacional, es que el banco estudiado entrega por contrato a los empleados y a las empleadas el beneficio de almuerzo, el cual es costeado totalmente por el empleador. Aunque los empleados $y$ las empleadas no tienen turnos para almorzar, todos tienen derecho a hacerlo y es por ello que el banco dispone de lugares para que su personal pueda comer.

En la casa matriz del banco existen dos espacios destinados para proveer alimentación a quienes trabajan ahí: uno de estos es de uso exclusivo de quienes detentan altos cargos gerenciales en la institución (el cual no fue estudiado); al otro pueden acceder la totalidad de quienes trabajan en la casa matriz, incluidos empleados y empleadas de sucursales regionales de paso en Santiago y personal subcontratado. La existencia de estos dos espacios de alimentación muestra cómo la comensalidad en una institución se da segmentada según las jerarquías institucionales y reflejan por lo tanto, la organización interna de la empresa (Grignon, 2001).

Por su parte, el comedor de la empresa de acceso general corresponde a una instalación donde se prepara comida fría y caliente, la cual es servida detrás de un mostrador o dispuesta en buffet para el autoservicio. La capacidad máxima de personas empleadas que pueden ser atendidos simultáneamente es de 300 personas $y$ en total el comedor utiliza un espacio físico de $582 \mathrm{~m}^{2}$, los cuales se dividen en 3 sectores: sector cocina $\left(158 \mathrm{~m}^{2}\right)$, sector línea de distribución $\left(112 \mathrm{~m}^{2}\right)$ y sector comedor $\left(312 \mathrm{~m}^{2}\right)$. Las personas empleadas cuentan con tarjetas electrónicas, las cuales sirven para entrar al sector de distribución activando un torniquete. Este mecanismo muestra las dinámicas de inclusión y exclusión propia de la comensalidad institucional (Grignon, 2001), dado que solo pueden compartir una mesa quienes forman parte del banco, quedando excluida cualquier persona que no tenga el pase institucional.

El servicio de comida es provisto por una empresa externa y el horario de atención cubre el almuerzo que se extiende entre 12:00 a 16:30 horas. Al ingresar hay tres estaciones de comida según el tipo de menú (criollo, vegetariano $y$ gourmet), los cuales son desplegados en una pantalla que está a la entrada del comedor. Al interior del comedor, las mesas cuentan con distintas dimensiones (3, 6 y 8 sillas a cada lado respectivamente) y con los mismos elementos sobre estas (limón, aceite de oliva, sal y un dispensador de servilletas). Cabe mencionar que además hay un horno microondas (que tiene poco uso) y dos televisores permanentemente prendidos que sintonizan noticias.

Las prácticas de comensalidad en el comedor se ven moldeadas por regulaciones institucionales, que influyen en lo que Claude Fischler (1995) denomina reglas extrínsecas de alimentación, principalmente aquellas referidas al tiempo y el lugar físico. En efecto, un entrevistado del área de RR.Hн., al que se llamara 
Pedro, describe el tiempo de almuerzo de las personas empleadas como un derecho y además una instancia necesaria que permite poner una pausa en la jornada laboral, pudiendo así compartir con colegas. Esta percepción es coherente con lo identificado en la literatura del rol de la alimentación en los ritmos de la jornada laboral ${ }^{4}$.

Además, se menciona que si bien el empleador no fija un tiempo máximo de almuerzo -reconociendo que las personas empleadas pueden demorarse entre 45 minutos a 1 hora para almorzar sin problemas- existe una suerte de confianza tácita que el empleado o la empleada no se tomará más de una hora para ello. El cumplimiento del tiempo de almuerzo se supervisa según cada departamento, ya sea si la persona se toma un tiempo mayor al estipulado o si tiene problemas para asistir al comedor por carga de trabajo. Como ejemplo de lo anterior, se ilustró esta situación con la experiencia de una cajera que trabajaba en comercio internacional, razón por la cual debía coordinar las conversiones de dinero según el horario en el cual iban abriendo las distintas bolsas de valores del mundo, la mayoría de estas con husos horarios distintos al chileno. Por esta razón, se le readecuó el tiempo del almuerzo.

Otro entrevistado de RR.HH., a quien se llamará Marcelo, señaló que es anormal tener que reconvenir a algún empleado o empleada por la duración de su almuerzo, cuestión que ocurría cuando la persona se había tomado 2 horas o más de colación. En específico, Marcelo comenta que existe una flexibilidad con relación al tiempo que utilizan las personas para almorzar, ya que la fiscalización sería únicamente necesaria cuando existe una falta notoria en relación con el tiempo asignado. Además, en caso de alguna demora evidente, Marcelo señala que se le pregunta a la persona empleada respecto a la razón del retraso.

Este fenómeno muestra que la alimentación dentro de un contexto laboral requiere de

4 Monjaret, A. (2002). L'alimentation au travail: bilan et perspectives. Consommations et Sociétés (2), L’Harmattan, pp. 7-21. la coordinación entre las exigencias productivas con aquellas propias de la reproducción de la vida (en este caso, comer). Los datos recién presentados revelan que las prácticas alimentarias son controladas y se encuentran constreñidas por las regulaciones implícitas y explícitas laborales, y que comportamientos desviados (utilizar más del tiempo estipulado para comer) reciben sanciones. La regulación sobre las prácticas de la alimentación en el trabajo es un ejemplo de disciplinamiento del cuerpo por parte del ejercicio del poder institucional (Foucault, 2002).

Es importante poner en diálogo las visiones institucionales antes descritas con la realización concreta de las prácticas de alimentación al interior del banco. Si bien, no existen turnos para ir al comedor, hay algunas labores específicas que imponen restricciones objetivas para decidir cuándo ir a almorzar (esto es obvio en el caso de empleados y empleadas que atienden público hasta las 14:00 horas). Junto con esto, se identificó que entre 12:00 a 13:00 horas y de 15:00 horas en adelante, el comedor tenía una afluencia de público menor a la experimentada de 13:00 a 15:00 horas (con mayor afluencia de 13:30 a 14:30 horas aproximadamente). Quienes almuerzan en los horarios con menor presencia de público, tienen facilidad para escoger donde situarse en el comedor y mayores posibilidades de elegir el menú que desean. Por otra parte, especialmente de 15:00 horas en adelante se notó una mayor flexibilidad referente al tiempo para almorzar, puesto que era más común que los grupos observados dedicaran 45 minutos para comer y 15 minutos a la sobremesa. A diferencia de estos grupos, las personas que llegaban en la hora punta (13:30 a 14:30 horas), veían restringidas sus posibilidades, tanto de elegir donde sentarse como de alargar sus horarios de almuerzo a través de la sobremesa.

Por lo anterior, generalmente estas personas tras terminar de llenar sus bandejas buscaban espacios que estuvieran disponibles, muchos de los cuales eran mesas que debían ser compartidas con otras personas. Asimismo, existió un apego a los 45 minutos de almuerzo (sin sobremesa), lo que se condice con la mayor 
rotación que tiene el comedor a esta hora, permitiendo a quienes iban entrando un lugar donde sentarse. Por lo tanto, junto a las reglas institucionales, las disposiciones del espacio físico que impone la empresa también determinan las prácticas de comensalidad y alimentación observadas.

Respecto a los espacios de almuerzo, Marcelo menciona que es poco frecuente que las personas prefieran almorzar en sus espacios de trabajo para avanzar con sus respectivas labores diarias. Por lo demás, el comedor institucional es el espacio establecido para poder almorzar, ya que por normativa sanitaria los empleados y las empleadas del banco no cuentan con espacios comunes en sus respectivos departamentos para calentar o prepararse comida. Este entrevistado señaló que durante los últimos años, el comedor ha tenido algunos cambios con el fin de modernizarlo $y$ de transformarlo en un lugar más agradable para quienes ahí almuerzan. En el 2014, se instalaron 2 televisores; en el 2015, se renovó el mobiliario de la cocina y en el año 2016, se dispusieron gráficas con paisajes de Chile en los muros. Además, aunque Marcelo señala que actualmente no existen políticas institucionales que promocionen un tipo particular de alimentación, sí se le exige al proveedor que entregue una dieta equilibrada y que entre las alternativas de almuerzo existan las dietas vegetarianas.

A partir de la preocupación por la decoración, entretenimiento (televisores) y mantenimiento del comedor (se observa un lugar limpio, iluminado, ventilado y con 3 distintos menús de opción), se puede sugerir que el espacio donde se almuerza expresa una dicotomía entre la necesidad de crear un lugar placentero para el comensal al tiempo que no puede escapar de las demandas propias de gestión de un comedor institucional (torniquetes a la entrada, puertas retráctiles que marcan el paso de un lugar a otro, espacios predeterminados donde están las comidas, mesas iguales, etc.). Ejemplo de lo anterior es la pantalla puesta a la entrada, la cual, en tanto solución tecnológica para informar a los asistentes, permite tener una idea rápida y concreta de lo que ese día el comedor tiene para ofrecer a las casi 300 personas que puede recibir.

Lo propio sucede con la regulación de los accesos, los cuales efectivamente limitan el uso del comedor solamente a quienes pueden activar los torniquetes con la tarjeta de identificación. Asimismo, las mesas dispuestas por el comedor son todas iguales y con los mismos elementos sobre ellas, lo que para efectos de comensalidad conlleva una mayor igualdad al compararla con mesas que permiten manifestar jerarquías por la cabecera de mesa (Fischler, 2011). De hecho, solo una vez se observó a alguien reacomodar las sillas para sentarse en la cabecera, en cuyo caso se hizo para permitir que el grupo entero pudiera almorzar en la misma mesa.

Por lo tanto, las regulaciones del banco y su infraestructura delimitan las condiciones sociotemporales de la comensalidad. Pero además de esto, es importante rescatar que las personas empleadas negocian con tales disposiciones al momento de almorzar. Esto lleva a recalcar la importancia de analizar las prácticas de comensalidad a la luz del contexto, atendiendo a las restricciones temporales propias de las jornadas laborales y del espacio físico donde se alimentan. Así, las prácticas de alimentación en el trabajo se encuentran delimitadas por las regulaciones institucionales, temporales y espaciales. Del mismo modo, la capacidad de agencia de los sujetos abre espacios para su personalización, negociación y adaptación.

Junto con esto, se pudo identificar una intencionalidad explícita por parte del banco en tratar de hacer del comedor un lugar más "agradable". Es decir, la alimentación parece ser un fenómeno emergente, con distintas dimensiones constitutivas. Es posible decir que existe un ideal regulatorio o un estándar normativo a cumplir relativo a la forma en que se ejecuta la alimentación. En otras palabras, no basta simplemente con hacerse cargo de dónde almuerzan los empleados y las empleadas, puesto que también existe una reflexión del banco referida a qué ambiente se proporciona y qué opciones alimenticias se ofertan. 
2) LAS PRÁCTICAS DE COMENSALIDAD

Y LAS CONVENCIONES SOCIALES

Respecto a las prácticas de comensalidad $y$ las convenciones sociales que se practican en el comedor del banco, se presentan los resultados en cuatro apartados: (i) constitución de grupos y límites, (ii) temas de sociabilidad y género, (iii) (des) estructuración y usos de la tecnología, y (iv) constreñimiento social y hábitos.

\section{$\diamond \quad$ Constitución de grupos y límites}

A diferencia de lo que sucede en un almuerzo familiar o con amigos, en el comedor del banco es frecuente que los empleados o las empleadas tengan que sentarse junto a personas desconocidas, incluso sin asientos de distancia. En esta situación, los comensales no tienen ningún poder de excluir a desconocidos de la mesa - salvo que las mesas estén completas- pero no por ello deja de operar una noción de intimidad ligada a la práctica de compartir una comida. Precisamente, es común que las personas tengan que pedir verbalmente permiso para poder integrarse a una mesa ya constituida. En este sentido, se observan límites espaciales que son reconocidos por los participantes y que dejan entrever un carácter simbólico de la cercanía.

Además, en caso de que dos grupos desconocidos entre sí tengan que compartir la mesa, el nivel de interacción es nulo, reduciendo la necesidad de comunicarse únicamente para pedir una servilleta o aderezo que no está al alcance inmediato. De este modo, se respetan los límites de interacción simbólicos, aun cuando físicamente exista una copresencialidad. De este modo, se pone de manifiesto que la comensalidad en el comedor estudiado demanda la preexistencia de un grupo de conocidos (Grignon, 2001).

Por otro lado, la cantidad de personas que configuran el número de comensales también juega un rol simbólico. En efecto, se observaron comensales que iban siempre juntos, personas sin un patrón establecido y aquellos que comen solos, tal como señala Heynig (2015), lo más común es que las personas vayan en grupos preexistentes a almorzar.
Parece que el almuerzo en el banco es un acto de sociabilidad que no busca establecer intimidad. Se observa que quienes comparten la mesa, lo hacen usualmente en grupos de 4 personas, siendo menos común observar parejas que comparten la mesa. Entre las parejas que almorzaban juntas, son sumamente atípicas las parejas de distinto sexo que asisten al comedor juntos, lo cual puede deberse a las valoraciones culturales que vinculan la acción de compartir una comida a una instancia cercana y eventualmente romántica (Fischler, 2011).

Junto con lo anterior, fue atípico ver personas que iban solas a almorzar, cuestión que se observó con más frecuencia en las horas de menor afluencia de público. Esto quizás puede deberse a las percepciones normativamente impropias que existen en torno a la alimentación solitaria (Giacoman, 2016), lo que por momentos fue notorio, por ejemplo, en distintas ocasiones con el fin de evidenciar que venían a sentarse en la misma mesa, diversos grupos de empleados se acercaron a la mesa de quien realizaba la observación señalando "te venimos a hacer compañía”. En otras palabras, se explicita la intromisión dentro del espacio simbólico de la persona que come sola, generando un momento de copresencialidad sin interacción.

\section{$\diamond \quad$ Temas de sociabilidad $y$ género}

Por otra parte, las interacciones verbales pueden ser definidas como una instancia de convivialidad, donde los empleados suelen conversar sobre lo ocurrido durante la jornada, hablan de sus familias y comentan las distintas noticias que aparecen en televisión. Es importante recalcar que se constató que la convivialidad no es sinónimo de que los temas tratados en el comedor siempre impliquen un consenso argumentativo.

Respecto a la comunicación y los temas abordados, se observó que en mesas compuestas de hombres, eran más comunes temáticas relacionadas al trabajo. En esta materia, los resultados están en consonancia con Mestdag (2005), ya que cuando se habla de trabajo se suele tematizar la experiencia diaria. Sin embargo, se observó una actitud restrictiva y 
reprobatoria entre los comensales cuando uno de ellos realizaba tareas laborales en la mesa (principalmente contestar mails). En mesas compuestas por mujeres, la temática del trabajo pasaba a un segundo plano, viéndose la preeminencia de relatos relacionados con hijos, historias personales $y$ anécdotas.

En este aspecto, la dinámica de una mesa mixta también resultaba ser diferente, especialmente si había más hombres que mujeres o viceversa. En el primero de los casos, generalmente eran los hombres quienes ponían y llevaban los temas. En el segundo caso, las mujeres también hablaban más, sin embargo, esto tendía a equipararse cuando el tema de conversación tenía relación con el trabajo. Cabe destacar que nunca se registró un comentario negativo sobre la comida del comedor, lo que hasta cierto punto facilita la construcción de un espacio para poder compartir $y$ hablar de otros temas. Por lo tanto, en el comedor observado, resulta evidente que compartir la mesa con compañeros y compañeras de trabajo constituye un momento de descanso e interacción, en el cual es posible socializar y distender la jornada laboral.

\section{$\diamond \quad$ (Des)estructuración y usos de la tecnología}

Se observó que quienes van solos a almorzar no manifiestan un patrón de desestructuración alimentaria ya que, al igual que las mesas grupales, comen 3 platos y 1 ensalada generalmente, modelo de alimentación que parece estar en gran medida condicionado por la oferta de platos que entrega el comedor institucional. La mayor diferencia radica en el tiempo que utilizan para comer (entre 35-45 minutos sin sobremesa) con respecto a quienes almuerzan en grupo (45 minutos-1 hora) y en el uso que hacen de la tecnología.

Sobre este último punto, quienes iban solos hacían un uso abierto del teléfono celular (llegando a utilizar audífonos para ver videos). Este fenómeno ha sido trabajado principalmente desde la sociología de la comunicación social. Sin embargo, utilizando un concepto extraído desde esa disciplina, se podría hablar de un efecto capullo, el cual se caracteriza por ser micro-lugares construidos a partir de infraestructura privada y que es controlada por el individuo (Ito, Okabe y Anderson, 2009). En este caso, el teléfono y los audífonos generan la infraestructura que aísla al individuo del resto del comedor.

En mesas grupales, el teléfono celular se usaba generalmente cuando las personas habían dejado de comer e interactuar entre sí. Por contrapartida, si el celular privatiza el acto alimentario en aquellos que acuden solos al comedor, el televisor tiene una función eminentemente social para las mesas grupales, pues está constantemente posicionando temas de conversación entre los asistentes. En cualquier escenario, mientras mayor era la persona, mayor era la concentración puesta en comer y menor en revisar dispositivos tecnológicos.

\section{$\diamond \quad$ Constreñimiento social y hábitos}

Las observaciones realizadas indican que compartir una mesa con conocidos en el comedor conlleva la realización de determinadas tareas comunes, principalmente aquellas que se relacionan con la regulación colectiva del tiempo de almuerzo, la obligación de interactuar verbalmente $y$ la ejecución de ciertos modales de mesa. Relativo a la regulación de los tiempos de alimentación, se pudo observar que cuando las personas empleadas iban acompañados al comedor, procuraban coordinar la ingesta de alimentos, con el fin de poder ir al mismo ritmo que los demás comensales. Esto fue una práctica observada incluso en aquellos casos que las personas no tenían la misma cantidad de platos (o siquiera el mismo tipo de menú).

Asimismo, en línea con lo descrito por Giacoman (2016) sobre el esfuerzo que hacen los compañeros y las compañeras de trabajo para almorzar juntos, también se registró la práctica de terminar el almuerzo y levantarse de la mesa de manera conjunta, aun cuando ciertos miembros del grupo habían llegado con retrasos de 15 minutos, lo cual había impedido que existiera la misma coordinación entre los comensales. De hecho, en estos casos muchas veces parte de los comensales ya habían terminado de comer $y$ sin embargo, esperaban a que su compañero o compeñera terminara de 
almorzar. Lo mismo fue observado en grupos de más de 6 personas, donde la regulación temporal y de los ritmos de la ingesta resultaba complicada por el número de comensales, puesto que las personas tendían a interactuar con aquellos que estaban más próximos.

En materia de modales de mesa, en primer lugar, fue posible observar que las formas de comer solitarias generalmente mostraban una menor rigurosidad en el despliegue de ciertos hábitos en comparación a las mesas grupales. En ese sentido, las observaciones muestran que la comensalidad en el comedor implica un mayor apego al uso de cubiertos, a no levantar tazones de sopa o recipientes de postres para comerlos, mantener la boca cerrada cuando se consumen alimentos y una evidente prolijidad al momento de evitar que la comida se cayera cuando era ingerida. Profundizando en esto, la observación de ciertas prácticas específicas - elevar la voz, sonarse en la mesa y hablar de temas personales entre otras - sugieren una vía de acceso a estudiar el entrecruce entre prácticas privadas en un espacio público o institucional (Lindén y Nyberg, 2009).

Por otro lado, se reafirma lo señalado por Devine et. ál (2007), puesto que el contexto institucional analizado influye en las porciones debido a que hay alimentos que se pueden repetir (bebidas, ensaladas y sopas) y otros que no, como es el caso de los platos de fondo. El género también tiene un efecto moderador en la ingesta, constando que los hombres comían porciones más grandes que las mujeres.

Siguiendo a Goffman (1999), por la forma en cómo se ejercen, podríamos decir que los modales de mesa son vehículos de signos que proyectan una definición de la situación de comensalidad, la cual en estudios posteriores podría ser profundizada a través de una contextualización jerárquica del espacio estudiado. Precisamente, los comensales en el comedor a través de los modales de mesa escenifican un "consenso de trabajo", un entendimiento práctico de las normas que atemáticamente debiesen seguir en la mesa, considerando además, como lo señala el autor, que la interacción cara-cara conlleva una "influencia recíproca de un individuo sobre las acciones del otro" (Goffman, 1999, p. 27).

\section{CONCLUSIONES}

La investigación exploratoria expuesta en el presente artículo ha permitido resaltar la cualidad social de la alimentación, dando cuenta de las prácticas de comensalidad de los empleados de un banco en Santiago de Chile. Junto con esto, la información recabada demuestra que la aproximación sociológica a la alimentación es una vía de acceso privilegiada al estudio de interacciones laborales, cuestionando así los postulados que naturalizan la alimentación como un hecho privado, biológico e individual.

Los resultados del estudio indican que este se encuentra en concordancia con la literatura disponible en cuanto a la alimentación en el trabajo como un momento de sociabilidad. Sin embargo, la contribución de este trabajo es proporcionar detalles que permiten identificar que las prácticas alimentarias de los empleados y las empleadas en tanto propias de la vida cotidiana, se ven estructuradas según las regulaciones temporales y espaciales del banco, sin que ello implique una incapacidad para adaptarse y negociar con tales disposiciones, lo que refleja su capacidad de agencia. Esto enfatiza la importancia de analizar cómo los contextos materiales y no materiales de la institución pueden influir al momento de la alimentación. Asimismo, las regulaciones $y$ discursos institucionales en torno a la alimentación permiten identificar que la alimentación es vista como un estándar normativo a cumplir que demanda más que solamente proveer de un espacio físico para almorzar.

Por otra parte, las observaciones evidencian que el compartir una mesa, incluso entre desconocidos, configura una relación de cercanía circunstancial y que posee límites simbólicos (como el silencio); sin embargo, para que se activen las reglamentaciones que subyacen a la comensalidad es necesaria la preexistencia de un grupo de conocidos. También, fue posible establecer que la comensalidad conlleva la realización de tareas colectivas ejemplificadas en una regulación temporal sincronizada entre 
los comensales, interacciones verbales que promueven un momento de sociabilidad y la puesta en escena de un conjunto particular de modales de mesa.

Futuros estudios sobre comensalidad institucional debiesen profundizar en la influencia que pueden tener las jerarquías internas de la institución sobre las interacciones de los comensales. De igual forma, es necesario seguir investigando hasta qué punto los contextos institucionales estandarizan las prácticas de comensalidad y cómo la alimentación puede ser un foco de sociabilidad entre compañeros y compañeras, más aún cuando se considera que parte relevante de la vida se pasa en el trabajo.

\section{REFERENCIAS}

Aymard, M., Grignon, C. y Sabban, F. (1993). A la recherche du temps social. En Aymard, M., Grignon, C., Sabban, F., Le temps de manger. Alimentation, emploi du temps et rythmes sociaux (pp. 1-40). Paris, France: Éditions de la Maison des sciences de l'homme. IsBN 2738004709.

Departamento de Estudios Dirección del Trabajo. (2014). Octava Encuesta Laboral ENCLA. Chile, Dirección del Trabajo. Recuperado de http://www.dt.gob. cl/documentacion/1612/w3-article108317.html

Devine, C., Nelson J., Chin, N., Dozier, A. y Fernandez, A. (2007). "Pizza Is Cheaper Than Salad": 'Assessing Workers' Views for an Environmental Food Intervention. Obesity, 15, pp. 57S-68S. ISBN 1930-739X.

Dirección del Trabajo. (1996). Ordenanza 2947. Chile. Recuperado de http://www.dt.gob. cl/legislacion/1611/w3-article-88474.html

Dirección del Trabajo. (2016a). Centro de Consultas Laborales. Chile. Recuperado de http://www.dt.gob.cl/consultas/1613/ w3-propertyvalue-22100.html

Dirección del Trabajo. (2016b). Código del Trabajo. Chile: Dirección del Trabajo. Recuperado de http://www.dt.gob.cl/legislacion/1611/articles-95516_recurso_1.pdf

Douglas, M. (1972). Deciphering a Meal. Daedalus, 101 (1), pp. 61-81. IsBN 00115266
Enets. (2009). Primera Encuesta Nacional de Empleo, Trabajo, Salud y Calidad de Vida de los Trabajadores y Trabajadoras en Chile. Chile, Ministerio de Salud. Recuperado de http://www.isl.gob.cl/wpcontent/uploads/2011/09/Informe-FinalENETS-Interinstitucional.pdf

Fischler, C. (1995). El (h)omnívoro: el gusto, la cocina y el cuerpo. Barcelona: Anagrama, pp. 175-218. IsBN 8433913980

Fischler, C. (2011). Commensality, society and culture. Social Science Information, 50 (3-4), pp. 528-548. IsBN 14617412.

Foucault, M. (2002). Vigilar y castigar: Nacimiento de la prisión. Buenos Aires: Siglo xxI, p. 314. ISBN $987987014 \mathrm{X}$

Giacoman, C. (2016). The dimensions and role of commensality: A theoretical model drawn from the significance of communal eating among adults in Santiago, Chile. Appetite, 107, pp. 460-470. IsBN 0195-6663.

Giacoman, C., Leal, D. y Rivera, V. (2017). Daily rhythms of eating in Santiago, Chile. British Food Journal, 119(6), pp. 11891201. IsBN 0007-070X.

Goffman, E. (1999). La presentación de la persona en la vida cotidiana. Buenos Aires: Amorrortu Editores, pp. 13-28. ISBN 9505180292.

Grignon, C. (2001). Commensality and Social Morphology: An essay of tipology. En P. Scholliers, Food, Drink and Identity. (pp. 23-33) Oxford: Berg, IsBN 9781859734612.

Heynig, E. (junio de 2015). Alimentación, comensalidad $y$ factores de riesgo psicosociales en el trabajo. El comer de los trabajadores de un hospital público en Chile. Ponencia en el IV Congreso Internacional del Observatorio de la Alimentación. Barcelona: oDELA.

Ito, M., Okabe, D. y Anderson, K. (2009). Portable objects in three global cities: The personalization of urban places. En Ling, R., Campbell, S. The reconstruction of space and time: mobile communication practices. (pp. 67-87). Nuevo Brunswick: Transaction Publisher. ISBN 9781412808095. 
Kniffin, K. M., Wansink, B., Devine, C. M. y Sobal, J. (2015). Eating Together at the Firehouse: How Workplace Commensality Relates to the Performance of Firefighters. Human Performance, 28, pp. 281-306. IsBN 1532-70435.

Lindén, A. L. y Nyberg, M. (2009). The workplace lunch room: an arena for multicultural eating. International Journal of Consumer Studies, 33, pp. 42-48. ISBN $1470-6431$.

Mestdag, I. (2005). Disappearance of the traditional meal: Temporal, social and spatial destructuration. Appetite, 45, pp. 62-74. ISBN 0195-6663.

Monjaret, A. (2002). L'alimentation au travail: bilan et perspectives. Consommations et Sociétés, (2), L’Harmattan, pp. 7-21. ISBN 2747515788.

ocDe. (2017). Hours Worked Indicator. овсD Employment Outlook, París. Recuperado de https://data.oecd.org/emp/hoursworked.html

огт. (2012). Un enfoque integral para mejorar la alimentación y nutrición en el trabajo: Estudio en empresas chilenas y recomendaciones adaptadas. Chile. Recuperado de http://www.ilo.org/santiago/publicaciones/wcms_201138/lang-es/ index.html

Shove. E, Pantzar. M. y Watson. M (2012). The Dynamics of Social Practice. Londres: SAGE, p. 208. ISBN 0857020439

Shove. E, Trentmann. F. y Wilk. R. (2009). Time, Consumption and Everyday Life: Practice, Materiality and Culture. Nueva York: Berg. IsBn 1847883648.

Simmel, G. (1986). Sociología de la comida. El Individuo Y la Libertad: Ensayos De Crítica De La Cultura. Barcelona: Península, pp. 263-270. IsBN 9788483074152
Sobal, J., Bove, C. y Rauschenbach, B. (2002). Commensal careers at entry into marriage: establishing commensal units and managing commensal circles. The Sociological Review, 50 (3), pp. 378-397. isbn 09275459.

Southerton, D. (2003). 'Squeezing Time' Allocating Practices, Coordinating Networks and Scheduling Society, Time \& Society, (12) 1, pp. 5-25. ISBN 14617463.

Southerton, D. (2013). Habits, Routines and Temporalities of Consumption: From Individual Behaviours to the Reproduction of Everyday Practices, Time \& Society, (22) 3, pp. 335-355. IsBN 14617463.

Stewart, A. J. y Wahlqvist, M. (1985). Effect of Shiftwork on Canteen Food Purchase. Journal of Occupational and Environmental Medicine, 27 (8), pp. 552 554. ISBN 15365948.

Takagi, K. (1972). Influence of shift work on time and frequency of meal taking. Journal of Human Ergology, 1, pp. 195205. ISBN 18843964.

Thelen, T. (2006). Lunch in an East German Enterprise-Differences in Eating Habits as Symbols of Collective Identity. Zeitschrift für Ethnologie, 131 (1), pp. 51-70. ISBN 00442666.

Valentine, G. (2002). In-corporations: Food, Bodies and Organizations. Body \& Society, 8 (2), pp. 1-20.

Waterhouse, J., Buckley, P., Edwards, B. y Reilly, T. (2003). Measurement of, and Some Reasons for, Differences in Eating Habits Between Night and Day Workers. Chronobiology International, 20 (6), pp. 1075-1092. IsBN 1525-6073.

Fecha de ingreso: $12 / 11 / 2017$ Fecha de aprobación: 26/06/2018 